\title{
Modèle hydrosédimentologique des écoulements hyperconcentrés d'un petit torrent des Alpes du sud
}

\author{
Ana Luiza Borges*, Sylvie Brochot, Maurice Meunier \\ CEMAGREF, Division Protection contre les Erosions, \\ Domaine Universitaire, BP 76, 38402 St-Martin d'Hères Cedex, France
}

\section{Introduction}

La détermination des transports solides revêt dans de nombreux bassins versants torrentiels une importance stratégique du fait de leurs conséquences: dépôts, affouillements, divagations, comblement de retenues... Il est donc souhaitable de disposer d'un modèle adapté

Dans le domaine de l'hydraulique fluviale, la connaissance, quoiqu'encore incomplète, est suffisamment maîtrisée pour que des modèles de transports solides aient été mis au point. Malheureusement, il n'est pas envisageable d'en extrapoler l'application au domaine torrentiel du fait de la complexité des phénomènes observés dans les bassins versants concernés. Parmi les facteurs de cette complexité, on peut citer les suivants :

- le débit solide est suffisamment important pour influencer la hauteur de l'écoulement; on ne peut plus l'assimiler à la hauteur d'eau, comme l'ont montré les expériences de Smart et Jaeggi (1983), à partir d'une pente de 7 à $8 \%$; - les versants d'un bassin torrentiel fournissent souvent la majeure partie des matériaux transportés. On ne peut donc plus considérer que ces derniers ne proviennent que du lit du torrent ;

- suivant le type et l'importance du débit solide, la nature des écoulements varie, (lave torrentielle, charriage hyperconcentré, suspension hyperconcentrée, charriage fluvial, eau claire). Le premier problème est d'ailleurs souvent celui

(*) Actuellement à l'Instituto de Pesquisas Hidràulicas de Porto Alegre, UFRGS, Brésil. de déterminer la nature de l'écoulement auquel on a affaire (cet aspect est abordé dans l'article introductif de M. Meunier dans ce numéro de La Houille Blanche);

- les irrégularités morphologiques dans l'espace constituent un caractère dominant des torrents (pente, profil en travers, granulométrie). En outre, tout au long d'une phase de charriage torrentiel, le lit, lorsque l'on ne lui en supprime pas la possibilité, divague et ses caractéristiques morphologiques varient également très rapidement dans le temps.

La modélisation en hydraulique fluviale (par exemple RAHUEL et al., 1989, ou LABADIE et al., 1991), se base en général sur les équations de conservation de la masse et de la quantité de mouvement, reliées à des lois de transport. On traite à chaque instant les transferts entre une ou plusieurs couches d'échange et le flux solide. On représente les processus internes, à partir des notions classiques, comme les seuils de début de transport ou de déposition. en fonction des classes granulométriques. On introduit les lois des échanges entre les classes et entre les couches d'échange, etc... De tels modèles s'appuient sur des expériences au laboratoire et sont souvent utilisés pour simuler l'effet de crues pour de grands cours d'eau. On peut aussi construire des modèles similaires s'intéressant à l'évolution à long terme de la morphologie, en effectuant la simulation à une autre échelle de temps, par exemple annuelle (DI Silvio G., Peviani M., 1989, Di Silvio G., 1991).

Compte tenu de toutes les difficultés existant déjà pour la modélisation des transports solides en hydraulique fluviale et des complications prévisibles pour les torrents, il est apparu souhaitable de tenter une autre approche,

\section{An hydrosedimentologic model for hyperconcentrated flows of a small torrent in the southern Alps}

This paper expounds the principles of a global spatialized event model, fitted to torrent floods. It reproduces both water and sediment flows through the catchments. The sediment transport formulas are specific to steep gradients. Furthermore, a formula for the break up of the armour layer has been introduced and a formula describing the turn of the bed load transport into suspension too. At the end, the paper exposes briefly the basin characteristics and the measurement devices, and compares the results of the model with the observed data. 
consistant à représenter globalement le fonctionnement d'un bassin versant torrentiel, aussi bien pour la dynamique des biefs que pour celle des versants: on y traite les écoulements en fonction des caractéristiques moyennes des bassins versants.

Sur le plan épistémologique, on étend simplement au transport solide et à la morphologie, les conceptions déjà en usage en hydrologie pour traduire la réponse d'un bassin à un épisode pluvieux et simuler le routage des crues dans les biefs. Le modèle présenté ici comprend d'ailleurs un module de transformation pluie-débit, semblable à beaucoup d'autres, qui fournit les entrées au module de routage des débits liquides et solides dans le lit.

Les mesures des transports solides sont toujours très difficiles, mais, pour les torrents, celles des débits liquides le sont aussi. Les possibilités de validation des modèles sont donc rares. Le site des bassins versants expérimentaux de Draix (Alpes de Haute-Provence, France) a permis de disposer des données adéquates pour la validation du modèle hydrosédimentologique élaboré ; on a utilisé celles disponibles sur le torrent du Laval $\left(1 \mathrm{~km}^{2}\right)$, qui relève à l'évidence du domaine de l' hydraulique torrentielle : on y a mesuré des concentrations de matières en suspension de $400 \mathrm{~g} / 1$ après piégeage des éléments les plus grossiers dans une plage de dépôt; le volume écoulé au cours d'une crue peut comporter couramment $10 \%$ de volume solide.

Dans cet article sera exposée la conception détaillée du modèle; on présentera ensuite le site expérimental puis les résultats obtenus.

\section{Un modèlé hydrosédimentologique adapté aux torrents}

Le modèle est événementiel, concu pour fonctionner sur des crues torrentielles.

Il est déterministe dans son architecture générale (fig. 1).

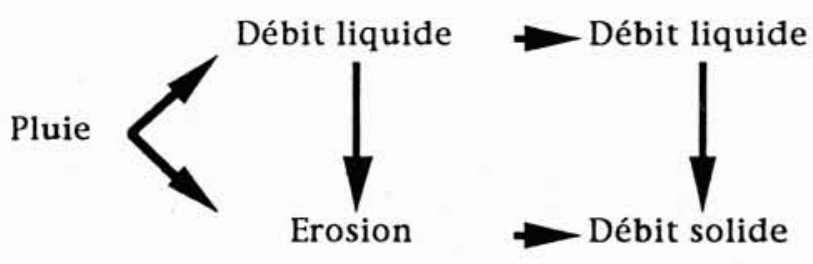

\section{VERSANTS}

BIEFS

1. Architecture générale du modèle.

Un logiciel, ETC (Erosion des torrents en crue), assure la continuité des écoulements au cours de leur traitement par les modèles élémentaires (représentés chacun par une flèche sur la figure 1). L'utilisateur doit sélectionner ces modèles élémentaires, parmi ceux offerts à son choix, en fonction des caractéristiques spécifiques du bassin versant torrentiel à traiter.

72

LA HOUILLE BLANCHE/N 3-1994
Pour tenir compte d'éventuelles hétérogénéités de la pluie et des caractéristiques physiques des bassins versants, ou pour mieux reproduire l'organisation du réseau hydrographique, le modèle peut être spatialisé : le bassin versant est alors représenté par une arborescence de versants ou sous-bassins et de biefs. ETC gère l'équation de continuité appliquée aux écoulements d'eau et de matériaux au travers de cette arborescence.

Comme l'hydraulique torrentielle est encore balbutiante, ETC a été concu comme un système évolutif, capable d'accueillir les nouvelles méthodes susceptibles d'être mises au point pour reproduire les comportements érosifs et hydrauliques torrentiels. (On illustrera d'ailleurs ci-après cette possibilité à propos de la modélisation de la transformation du charriage en suspension).

La conception générale des modèles pluie-débit et pluieérosion (sur les versants) ne sera pas explicitée ici ; les choix faits pour leur application au cas spécifique du bassin du Laval seront décrits dans le $\S 2$ de cet article.

\subsection{Routage du débit liquide dans les biefs}

On admet que les écoulements se font toujours en régime torrentiel, ce qui permet de s'affranchir de l'influence de l'aval et permet une résolution totalement différente des schémas classiques : la propagation de la crue est traitée en totalité pour chaque bief, et chaque bief est traité successivement de l'amont vers l'aval. On considère la crue comme une succession de régimes permanents. De même, les biefs sont des biefs uniformes, de pente constante.

La propagation de la crue liquide dans un bief est une simple translation sans déformation, ce qui est admissible pour les fortes pentes.

\subsection{Routage du transport solide dans les biefs}

Le transport solide est traité grâce à l'équation de continuité aux nœuds. Pour les biefs, l'organisation du module de routage du transport solide (MEUNIER M., 1987, LEFRANC P., 1990) est représentée sur la figure 2. Ce module traduit le fonctionnement des torrents, tel qu'il est assez couramment admis par les praticiens. Le débit solide entrant est d'abord comparé à la capacité maximale de transport :

- s'il est supérieur, le supplément est considéré comme devant se déposer. Il alimente ainsi un volume de sédiments accessibles à tout moment à l'érosion ;

— s'il est inférieur, l'écoulement s'alimente dans le volume de sédiments disponibles pour satisfaire sa capacité maximale de transport. Si cette réserve n'est pas suffisante, il y a possibilité d'érosion du lit du torrent lui-même par le biais d'une formule de dépavage.

Ce modèle étant concu pour représenter les comportements torrentiels, les formules de transport solide utilisées sont celles qui ont été obtenues à partir d'essais sur modèle réduit à forte pente :

- Formule de Smart et Jaeggi (SMART G.M., JAEGGI M.R., 1983)

$$
\Phi_{B . C h}=4 /(s-1)\left(D_{90} / D_{30}\right)^{0,2} I^{1,6} q\left(1-\tau_{c} / \tau\right)
$$




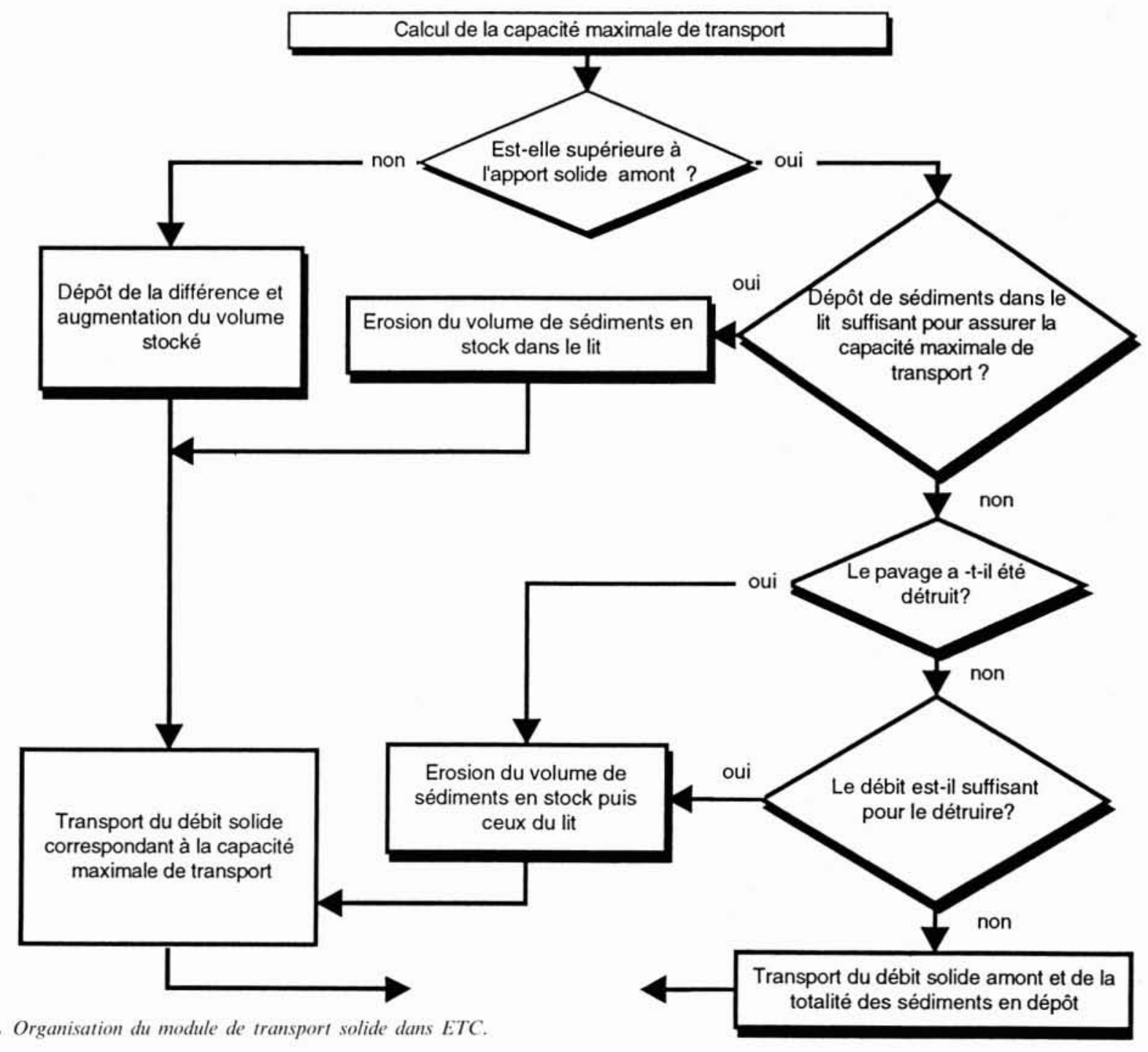

2. Organisation du module de transport solide dans ETC.

où $\Phi_{B, C h}$ est le débit solide adimensionnel en charriage, $s$ le rapport des masses volumiques entre ce débit solide et l'eau, $D_{90}$ et $D_{30}$ les quantiles de la courbe granulométrique du matériau transporté, $I$ la pente, $q$ le débit liquide par unité de largeur, $\tau$ la contrainte de cisaillement, et $\tau_{c}$ la contrainte de cisaillement adimensionnelle.

- Formule de Rickenmann (Rickenmann D., 1990)

$q_{B . C h} / q=12,6\left(D_{90} / D_{30}\right)^{0.2}\left(\rho \rho_{m} /\left(\rho_{s}-\rho_{m}\right)\right)^{1.6} I^{2}\left(1-q_{c r} / q\right)$

où $\rho_{m}$ et $\rho_{s}$ sont les masses volumiques respectives du mélange et du matériau solide, et $q_{B C h}$ est le débit solide de charriage par unité de largeur. Le début de mouvement est introduit ici à partir d'un débit critique $q_{c r}$ et non à partir de la valeur critique de la contrainte de cisaillement. L'intérêt de cette formule provient des expériences sur lesquelles elle a été calée, qui consistaient à étudier l'effet sur le transport solide d'une augmentation de densité du fluide par adjonction de matériaux fins.

- Formule de Lefort (LeForT P., 1991)

$Q_{B . C h} / Q=4,45\left(D_{90} / D_{30}\right)^{0.2}\left(\rho /\left(\rho_{s-} \rho\right)\right) I^{1.5}\left(1-\left(Q_{c r} / Q\right)^{0.375}\right)$
Cette formule, calée sur des essais sur modèle réduit en matériau granulaire, est valable pour une vaste gamme de pentes. Elle a été élaborée à partir des débits totaux $Q$ et $Q_{B \text {.Ch }}$, et non du débit par unité de largeur $q$. ce qui élude le problème souvent difficile du choix de la largeur à utiliser dans ces formules, ainsi que celui de la formule de perte de charge à utiliser.

Comme on le voit, l'effet de la courbe granulométrique est traduit par la présence du facteur $\left(D_{90} / D_{30}\right)$; ce modèle ne décompose done pas la courbe granulométrique en classes et ne traite pas les phénomènes qui lui sont reliés (masquage, tri granulométrique, formation du pavage), comme le font les modèles plus complets qu'on utilise en hydraulique fluviale.

Chaque formule de transport solide a son propre seuil de début de transport. Une formule supplémentaire a été utilisée pour traduire le dépavage, celle de Bathurst (BATHURST J.C., 1987) :

$$
q_{c p}=0,15 I^{-1.12 g^{0.5}} D_{50}^{1.5}
$$

oủ $q_{c p}$ est le débit unitaire de dépavage. On a donc la possibilité de séparer la loi d'érosion d'un lit pavé de celle des dépôts. 


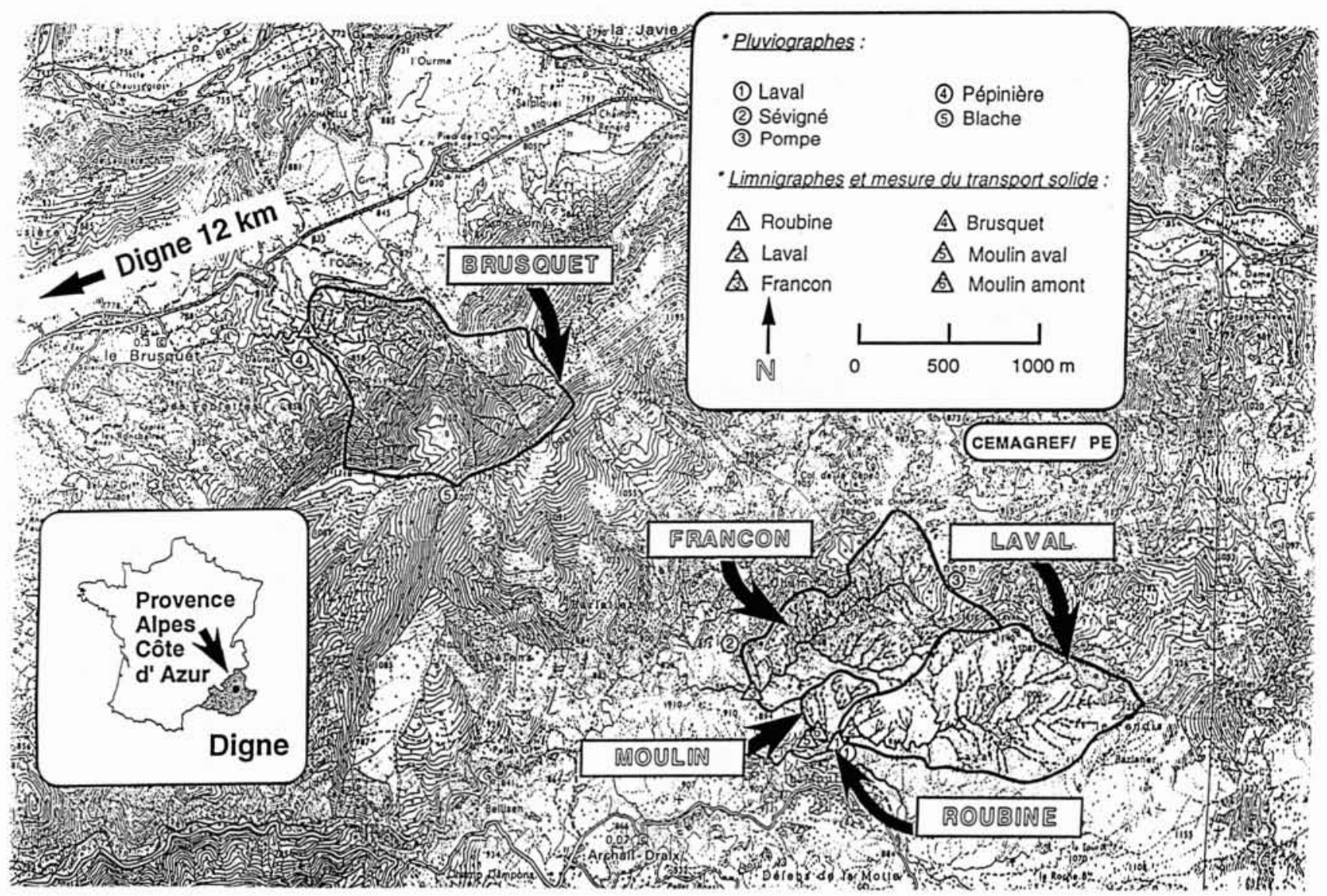

3. Plan de situation des bassins versants expérimentaux de Draix (Alpes de Haute-Provence, France).

\section{Le site expérimental du Laval à Draix et l'adap- tation du modèle}

Il est en général très difficile de vérifier la validité d'un modèle de transport solide à partir de données réelles. Les flux notamment sont très mal connus, et, souvent, on ne peut confronter les résultats du calcul qu'à des mesures ou observations limitées; comme l'état du lit (RAHUEL J.L., 1988, pour le Rhône, ou LiN H., 1993, sur un collecteur de réseau d'eaux usées).

Très différent est le cas du site des bassins versants expérimentaux de Draix (Alpes de Haute-Provence, France), pour lequel des mesures adéquates sont faites sur un torrent de moins de $1 \mathrm{~km}^{2}$, le Laval; elles ont donc permis de tester le modèle hydrosédimentologique décrit ci-dessus. Mais ce torrent présentant de solides particularités, il a fallu compléter celui-ci.

\subsection{Description succincte des bassins versants expérimen- taux de Draix et des données disponibles}

Les bassins versants de Draix se trouvent dans les Alpes du sud (fig. 3). Ils sont situés sur des terrains marneux et marno-calcaires très érodables: l'ablation moyenne interannuelle est de l'ordre de 130 à $160 \mathrm{t} / \mathrm{ha}$.an, valeur couramment observée sur de tels terrains (COMBES F., 1981,
Buffalo M., 1989). Ceci donne des écoulements torrentiels à très fort débit solide, pouvant aller jusqu'à des laves torrentielles. Le débit solide mesuré comporte deux termes: le volume déposé dans un piège à sédiments mesuré globalement, et le volume transité au travers d'un barrage filtrant, obtenu en échantillonnant la crue avec un préleveur automatique fonctionnant de manière discontinue. Le mode et les difficultés de fonctionnement des appareillages ont été décrits par CAMBON J.P. et al. (1990), le type et les difficultés de traitement des données obtenues par Mathys N. et Meunier M. (1988) ou Borges A.L. (1993).

Du point de vue hydrologique, les crues du Laval sont brèves (temps de réponse de l'ordre de 20 à 30 minutes). Les biefs (fig. 4) servent de transit aux écoulements qui arrivent des versants fortement chargés de sédiments; ils peuvent déposer une part non négligeable de leur charge dans les biefs ou, à l'inverse, éroder ces dépôts lors d'une autre crue. Par contre, le fond des biefs est rocheux (marne non altérée) et la capacité d'érosion est donc limitée aux stocks en place.

\subsection{Conception du modèle hydrosédimentologique du Laval}

L'application du modèle hydrosédimentologique décrit cidessus aux écoulements torrentiels du Laval se heurte à deux problèmes, celui des conditions aux limites amont, 


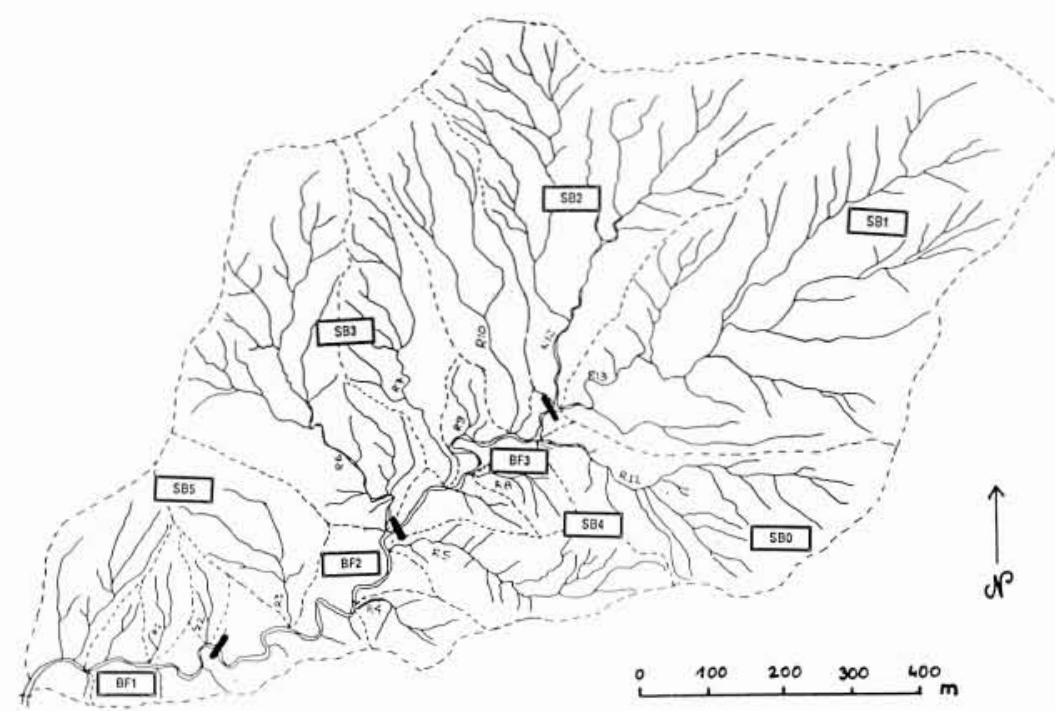

4. Bassin versant du Laval.

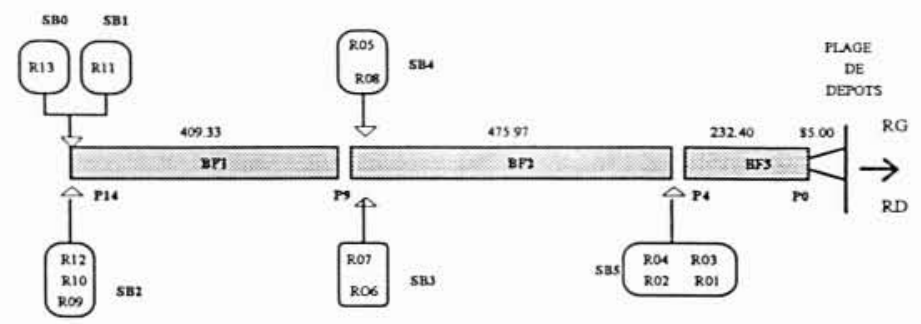

5. Représentation simplifiée du bassin du Laval.

problème général à toute modélisation, et celui de la nature des écoulements done de la possibilité de les traduire par des formules de transport.

\subsubsection{Conditions aux limites amont}

Le bassin versant est représenté sur la figure 4. Après analyse, le lit de la branche principale a été découpé en trois biefs, et les ravines ont été regroupées en six sousbassins (fig. 5), sur lesquels ont été calculées les entrées préalables au routage dans les biefs. En effet, celles-ci ne sont pas mesurées, et il faut donc commencer par les estimer.

Le modèle pluie-débit choisi pour représenter les crues est un modèle très simple ; il comporte pour la fonction de production, un seuil initial avant ruissellement ; sur la pluie nette ainsi calculée, on applique un coefficient de ruissellement constant. Ces deux paramètres variant d'un épisode à un autre, ce sont les paramètres optimaux qui ont été choisis lors de l'utilisation du modèle pour étudier les transports solides. Pour la fonction de transfert, un hydrogramme unitaire triangulaire de base égale à deux fois le temps de concentration $\left(T_{C}\right)$ a été utilisé. Le temps de concentration est obtenu à partir d'une formule adaptée de celle d'Ischbach pour les torrents de montagne (MEUNIER

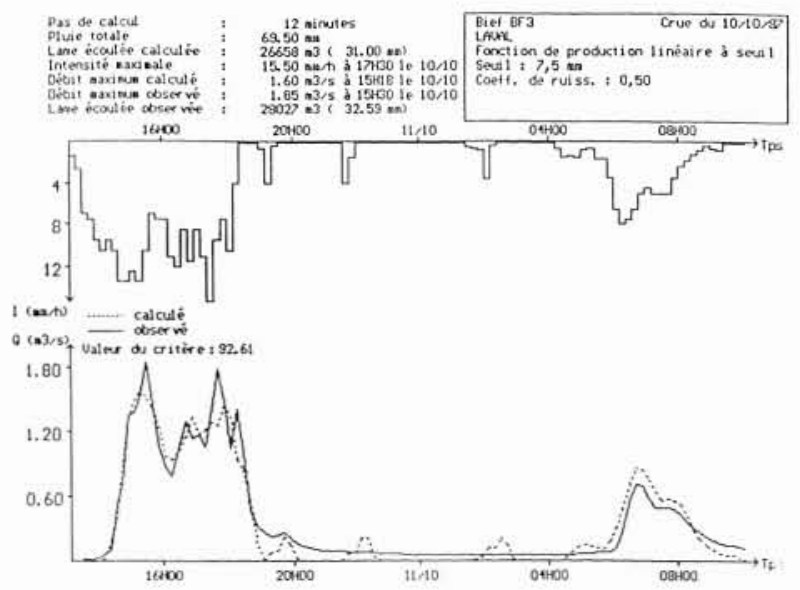

6. Simulation de la crue du 10/10/87 au Laval.

M. et Mathys N.,1989). La figure 6 donne un exemple de comparaison entre les débits liquides calculés et mesurés.

Le transport solide à l'entrée du modèle hydraulique a été obtenu de la manière suivante : la production totale de sédiments d'une petite ravine $\left(1330 \mathrm{~m}^{2}\right)$, appelée la 
Roubine, étant mesurée à chaque crue, on transfère ces mesures aux sous bassins du Laval, proportionnellement au produit (surface érodée * pente du talweg). Ce volume d'érosion est alors distribué dans le temps, proportionnellement au débit liquide à la sortie de chaque sous-bassin pour donner son sédimentogramme. De plus, comme la production de sédiments de la ravine mesurée est répartie en deux termes (volume déposé dans le piège à sédiments : $85 \%$; volume transité à l'aval de la grille du piège : $15 \%$ ), on conserve cette répartition à la sortie des sous-bassins du Laval.

Comme on le voit, on ne maîtrise pas réellement les conditions amont du modèle et les écarts en sortie entre les mesures et les calculs peuvent très bien provenir de cette non-connaissance. C'est un problème difficilement surmontable pour la plupart des torrents de montagne, pour lesquels des mesures constituent une denrée quasi introuvable.

\subsubsection{La nature des écoulements}

Les écoulements observés sur le Laval sont évidemment des écoulements hyperconcentrés: le rapport $\left(Q_{B} / Q\right)$ du débit solide au débit liquide varie entre quelques $\%$ et quelques dizaines de $\%$; après décantation dans la plage de dépôts, on a mesuré des concentrations allant jusqu'à près de $500 \mathrm{~g} / 1$ de matériaux fins, c'est-à-dire des concentrations correspondant aux écoulements observés sur les affluents du Fleuve Jaune (ZHANG J. et al., 1990). Ceci indique que les formules programmées dans ETC, établies à partir d'écoulements granulaires, ne sont pas forcément valables ici.

La répartition entre les matériaux transités et déposés est de plus très variable : de $15 \% / 85 \%$ sur la Roubine, elle passe à $60 \% / 40 \%$ à l'exutoire du Laval. Le matériau grossier se dégrade en effet très rapidement au cours du transport. Ceci a deux conséquences :

- Les formules utilisées dans ETC et indiquées ci-dessus ont été obtenues sur modèle réduit avec du matériau granulaire; en principe donc, ces formules expérimentales ne devraient pas bien représenter les écoulements du Laval composés pour moitié d'éléments fins (fig.7); seule celle de Rickenmann offre la possibilité de tenir compte de ces éléments fins. Elle possède donc un a priori favorable. Par ailleurs, on sait que ces formules expérimentales obtenues au laboratoire ont tendance à surestimer les transports réels, en général faute de sédiments disponibles pour l'érosion des lits.

- La dégradation rapide des matériaux grossiers en matériaux fins le long des biefs, ne peut être occultée. Elle a été prise en compte par le biais d'une loi de type exponentiel, obtenue en supposant que le taux de dégradation unitaire est proportionnel au taux d'éléments grossiers existant :

$$
Q_{B . G}=\alpha Q_{B} \quad \mathrm{~d} \alpha / \mathrm{d} L=-k \alpha
$$

où $Q_{B . G}$ est le débit solide des éléments grossiers, et $L$ est la longueur parcourue par les sédiments. Un calage sur les valeurs annuelles permet de donner à $k$ la valeur 0,0011 .

Pour modéliser les écoulements du Laval, on est donc conduit à assimiler :

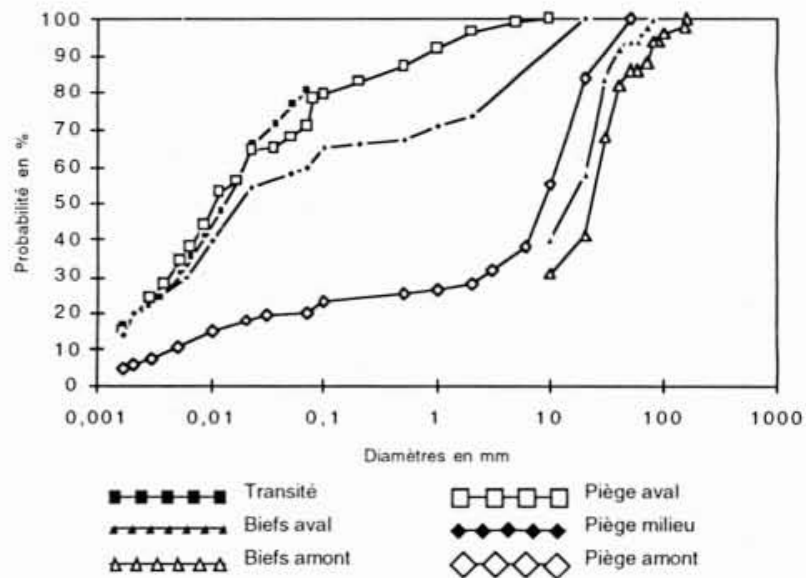

7. Courbes granulométriques des dépôts et du matériau transité au Laval.

- d'une part, le débit d'éléments grossiers au débit solide de charriage, donné par les formules expérimentales;

- d'autre part, le débit d'éléments fins au débit en suspension.

Ces assimilations n'ont rien d'évident a priori, car avec des écoulements hyperconcentrés, la séparation usuelle entre charriage et suspension perd de sa pertinence: le transport solide se fait en bloc, sans tri granulométrique notable quand on est à la capacité maximale de transport ; cela est d'autant plus vrai que le transport solide est important, donc qu'on se rapproche des laves torrentielles, pour lesquelles on sait que le tri granulométrique est inexistant. Le problème est différent lorsqu'on est proche du seuil d'entraînement.

Pour confronter les résultats des calculs aux mesures, on sera obligé d'admettre que le matériau déposé dans le piège à sédiments représente le charriage (hyperconcentré) et que le sédiment transité représente le complément, qu'on appellera suspension. Or, on sait qu'une part importante de sédiments fins est également déposée dans le piège ; on peut le voir sur la figure 7 , qui représente des courbes granulométriques des dépôts dans le piège à sédiments, celle du matériau transité, et celles des dépôts naturels dans certains biefs du Laval. Cette figure montre bien l'ampleur de l'hypothèse faite en assimilant le matériau piégé à du charriage.

Par ailleurs, pour être cohérent avec les formules de transport de charriage hyperconcentré introduites dans ETC, les courbes granulométriques des biefs seulement sont utilisées pour déterminer les quantiles $\left(D_{90}, D_{50}, D_{30}\right)$ nécessaires au calcul.

\section{Résultats}

\subsection{Données}

Les caractéristiques des biefs et sous-bassins sont données dans le tableau ci-dessous (tabl. I); la comparaison à des mesures ne peut être faite que pour les crues où les données sont suffisamment validées; or, notamment en raison du manque de fiabilité de la mesure des transports solides, on 
Tableau 1.

Caractéristiques des biefs et des sous-bassins.

SOUS-BASSINS

\begin{tabular}{|c|c|c|c|c|c|c|}
\hline $\begin{array}{l}\text { Dénomina- } \\
\text { tion des } \\
\text { sous-bassins }\end{array}$ & $\begin{array}{c}\begin{array}{c}\text { Surface } \\
\text { totale }\end{array} \\
\text { (ha) }\end{array}$ & $\begin{array}{l}\text { Surface } \\
\text { végéta- } \\
\text { liscéc } \\
\text { (ha) }\end{array}$ & $\begin{array}{c}\text { Surface } \\
\text { dénudée } \\
\text { (ha) }\end{array}$ & $\begin{array}{l}\text { Pente } \\
(\%)\end{array}$ & $\begin{array}{l}\text { Lon- } \\
\text { gueur } \\
\text { (m) }\end{array}$ & $(\mathrm{mn})$ \\
\hline SB0 & 7.3 & 1.7 & 5.6 & 38 & 490 & 7 \\
\hline SB1 & 22.3 & 8.0 & 14,33 & 34 & 889 & 14 \\
\hline SB2 & $20,4 \mathrm{~K}$ & 6,39 & 14,09 & 40 & 674 & 10 \\
\hline SB3 & 15,4 & 3,67 & 11,73 & 33 & 659 & 9 \\
\hline SB4 & 5.9 & 0.54 & 5.36 & 34 & 464 & 7 \\
\hline SB5 & 13.52 & 1.35 & 12,14 & 42 & 300 & 6 \\
\hline
\end{tabular}

BIEFS

\begin{tabular}{|c|c|c|c|c|c|c|c|}
\hline $\begin{array}{c}\text { Déno- } \\
\text { mina- } \\
\text { tion } \\
\text { des } \\
\text { hiefs }\end{array}$ & $\begin{array}{c}\text { Lon- } \\
\text { gueur }\end{array}$ & Largeur & Pente & D90 & D50 & D30 & $\begin{array}{c}\text { Coeffi- } \\
\text { cient de } \\
\text { Stri- } \\
\text { ckler }\end{array}$ \\
\hline $\mathrm{BF} 1$ & 410 & 3,8 & 6,0 & 64 & 19 & 10 & 13 \\
\hline $\mathrm{BF} 2$ & 476 & 4,5 & 4,0 & 40 & 17 & 9 & 14 \\
\hline $\mathrm{BF} 3$ & 233 & 4,3 & 4,5 & 31 & 15 & 6 & 15 \\
\hline
\end{tabular}

Tableau 2.

Caractéristiques des crues servant au calage.

\begin{tabular}{|c|c|c|c|c|c|c|c|c|}
\hline \multirow[b]{2}{*}{ Date } & \multirow[b]{2}{*}{$\begin{array}{l}\text { Pluie } \\
\text { totale } \\
\text { (mm) }\end{array}$} & \multirow[b]{2}{*}{$\begin{array}{l}\text { Inten. } \\
\text { sitê en } \\
1 \mathrm{mn}\end{array}$} & \multirow[b]{2}{*}{$\begin{array}{l}\text { L.ame } \\
\text { écoulée } \\
(\mathrm{mm})\end{array}$} & \multirow[b]{2}{*}{$\begin{array}{c}\text { Débit } \\
\text { maxi: } \\
\text { mal } \\
\\
\left(\mathrm{m}^{3} / \mathrm{s}\right)\end{array}$} & \multirow[b]{2}{*}{$\begin{array}{c}\text { Erosion } \\
\text { transitée } \\
\text { mesurée } \\
\text { par } \\
\text { préléve- } \\
\text { ment } \\
\text { (m3) }\end{array}$} & \multirow[b]{2}{*}{$\begin{array}{c}\text { Erosion } \\
\text { déposée } \\
\text { densité } \\
1.7 \\
\text { (m3) }\end{array}$} & \multicolumn{2}{|c|}{$\begin{array}{c}\text { Erosion totale } \\
\text { au Laval }\end{array}$} \\
\hline & & & & & & & $\begin{array}{l}\text { mesurée } \\
\text { au Laval }\end{array}$ & $\begin{array}{c}\text { Iransférée } \\
\text { depuis les } \\
\text { nesures de } \\
\text { Ia } \\
\text { Roubine }\end{array}$ \\
\hline $24 / 08 / 87$ & 46,2 & 90 & 6.0 & 2,708 & 279 & 210 & 1096 & 1423 \\
\hline $20 / 08 / 88$ & 25.2 & 78 & 4.8 & 1.63 & 258 & 65 & 794 & 723 \\
\hline $19 / 10 / 88$ & 73,7 & 66 & 35.9 & 2,88 & 645 & 395 & 2381 & 1367 \\
\hline
\end{tabular}

n’a trouvé que trois crues pour lesquelles tous les appareils ont fonctionné de manière satisfaisante, et même pour l'une d'entre elles (24/8/87), l'hydrogramme a été reconstitué ; les mesures de transport solide sont par contre très bonnes. Ces trois crues ont donc été utilisées pour la confrontation entre la mesure et les calculs ; le tableau 2 en indique les caractéristiques, avec les chiffres estimés (résultat des reconstitutions) écrits en italique.

Comme on le constate, ces trois crues ont un débit maximal très élevé. La simple observation de ces données montre que le rapport du volume transité au volume déposé à l'exutoire (dont on a vu qu'il est égal à 1,5 en moyenne) n'est absolument pas constant d'une crue à l'autre: il possède ici les valeurs suivantes: $2 / 6,2$ et 2,5 . Comme le calcul prévu dans notre modèle est basé sur le rapport moyen de 1,5 , il ne faut pas s'attendre à des résultats excellents sur ce point. Ce tableau montre aussi que le matériau solide entrant (érosion totale au Laval transférée depuis les mesures de la Roubine) est du même ordre de grandeur que le flux sortant du bassin du Laval (érosion totale mesurée); il est inférieur à deux reprises, mais l'inverse est également vrai (crue du 24/8/87). Ces différences proviennent en principe essentiellement des phénomènes de dépôts et reprises dans les biefs du Laval ; c'est d'ailleurs aussi cela que le modèle est censé représenter.

\subsection{Simulation des crues liquides}

La seule variable sur laquelle il est possible de comparer les résultats du modèle aux mesures sur toute la durée de la crue est le débit. Les résultats sont présentés sur la figure 8 .

Cette figure montre que l'accord entre les débits liquides calculés et mesurés est suffisant pour qu'on puisse admettre que le calcul des transports solides donne le même résultat, qu'on le fasse à partir des débits liquides mesurés ou calculés.

\subsection{Confrontation aux mesures de transport solide}

Pour les deux premières crues, les calculs ont montré que les dépôts dans les biefs étaient augmentés au passage de la crue ; l'initialisation des dépôts disponibles n'a donc pas d'influence sur les résultats. Mais il en est tout autrement pour la crue du 19/10/88, pour laquelle, dans l'ignorance où nous sommes de l'importance des stocks initiaux, deux calculs ont été effectués: le premier, en supposant l'absence de stocks disponibles, le second (noté 19/10/88 bis) en supposant au contraire que ces stocks sont très importants et que l'érosion par la crue peut s'exercer sans limite.

La figure 9 confronte les valeurs des volumes charriés, calculés par le modèle, d'une part, et les volumes déposés dans le piège, mesurés, d'autre part. Compte tenu des incertitudes bien connues concernant la validité de l'uti-

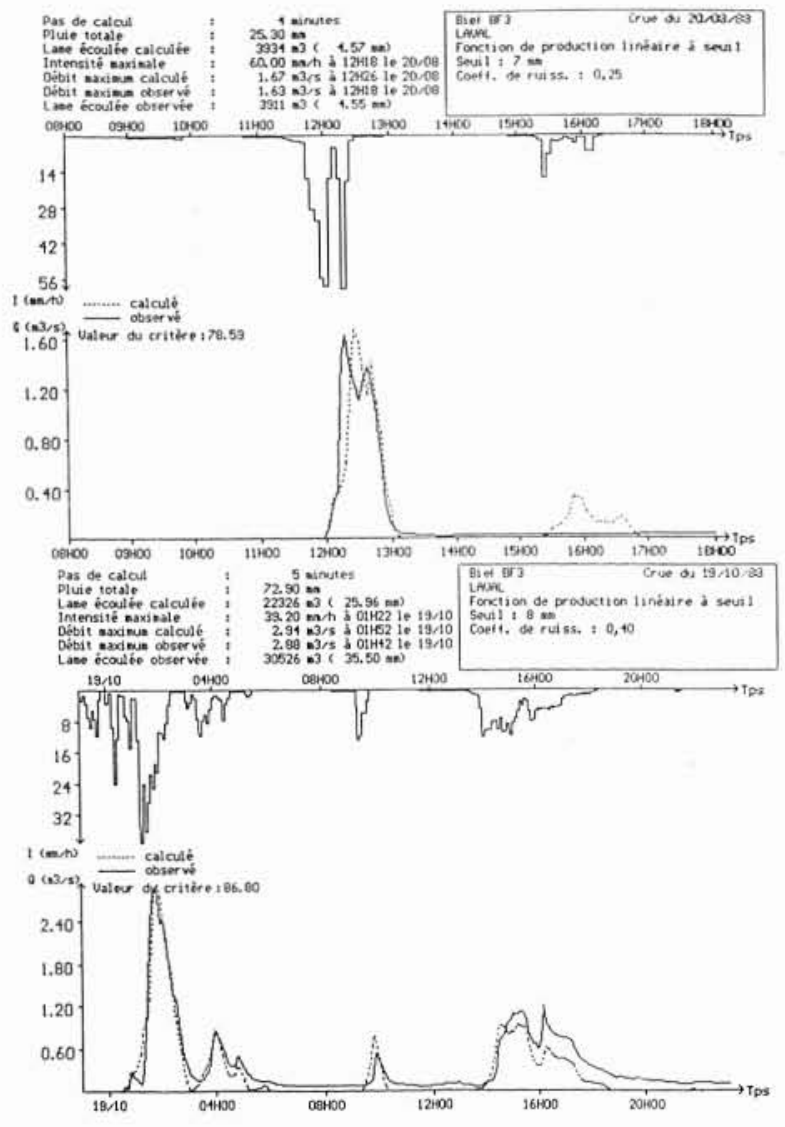

8. Crues du 20/8/88 et du 19/10/88. 


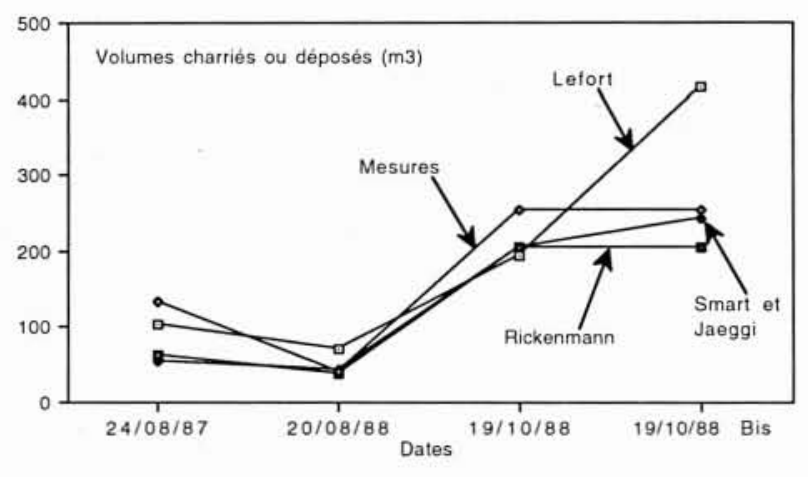

9. Confrontation des volumes transportés par charriage calculés à la mesure des dépôts dans le piège.

lisation des formules de transport solide en charriage, et leur application en particulier au bassin versant du Laval, on peut être surpris de leur excellente performance sur les trois crues utilisées dans cette confrontation aux mesures. On voit également que la formule de Lefort a été limitée dans son calcul par la disponibilité en sédiments et que si on lui donne la possibilité d'en éroder plus (19/10/88 bis), elle en transporte une quantité impressionnante. Cela montre l'importance qu'il y aurait à connaître le stock qui était réellement disponible dans les biefs.

Pour les suspensions (fig. 10), l'accord est moins parfait : le modèle sous-estime les volumes transités, ce qui est logique, puisque, pour ces trois crues, le rapport transité/déposé est supérieur à la valeur moyenne de 1,5. On remarque également que les volumes de suspension calculés reproduisent logiquement l'évolution des volumes de charriage calculés. Enfin, dans le cas où un stock de sédiments important est disponible dans les biefs, la formule de Lefort donne alors un volume de suspension plus adapté, conséquence directe du fait qu'elle a surestimé le volume transporté par charriage.

Conséquence directe de la sous-estimation des volumes en suspension, le modèle (fig. II) surestime pour ces trois crues, les dépôts dans les biefs, sauf pour l'épisode noté $19 / 10 / 88$ bis, pour lequel la formule de Lefort en érode juste ce qui est nécessaire. Mais il faut rappeler que les dépôts ne sont ici qu'estimés et non réellement mesurés. On voit qu'un suivi de l'évolution de ces dépôts, avec leurs mesures, permettrait de valider de manière très fructueuse les résultats du modèle.

En conclusion, la comparaison pour ces trois fortes crues, entre le modèle et les calculs, montre que :

- les formules de calcul du charriage hyperconcentré sont curieusement excellentes; elles sous-dimensionnent légèrement le volume recueilli dans la plage de dépôts;

- cette comparaison est rendue hasardeuse par l'absence de mesure des débits solides entrant dans les biefs, et des dépôts existants. Les calculs faits pour la crue du 19/10/88 montrent bien la différence obtenue suivant qu'on a au départ un stock disponible important ou nul;

- le modèle de dégradation du charriage en suspension sous-estime la suspension pour ces trois crues. Il faut plus de mesures pour qu'on puisse adopter définitivement la valeur 0,0011 du paramètre de ce modèle.

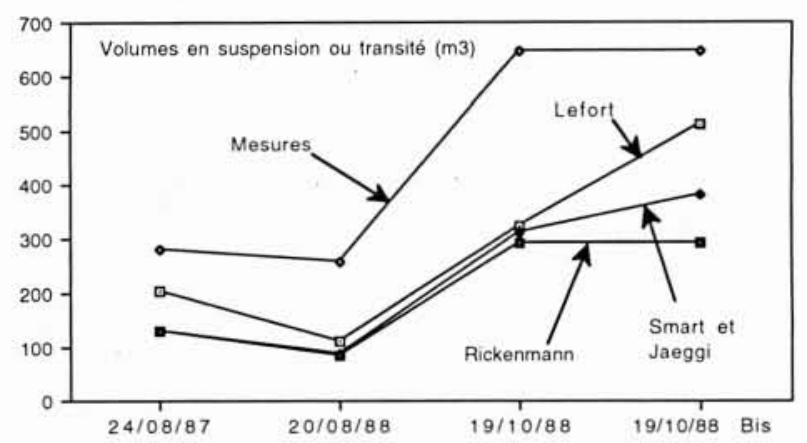

10. Confrontation des volumes transportés en suspension calculés aux volumes transités mesurés.

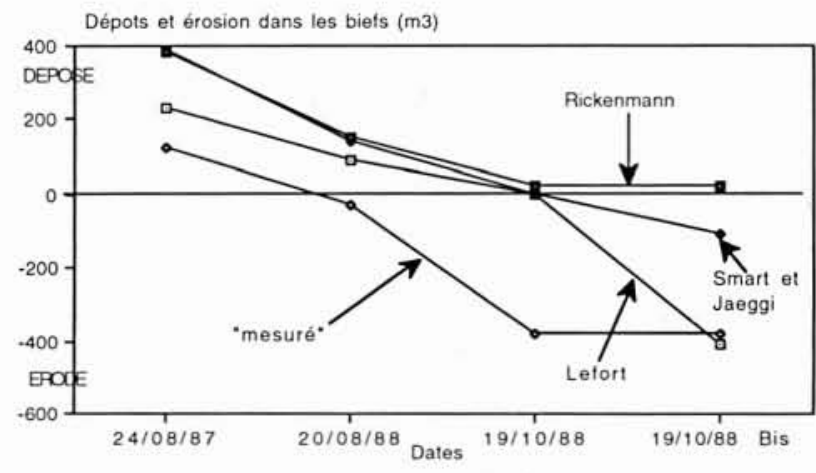

11. Confrontation des volumes déposés ou érodés dans les biefs, calculés, aux volumes estimés.

\subsection{Tentative de validation}

Les confrontations aux mesures présentées au paragraphe ci-dessus, présentent l'inconvénient d'être ponctuelles, dégagées de l'histoire des dépôts et reprises avant chaque crue, lesquels n'étaient d'ailleurs pas particulièrement observés à cette époque. Par ailleurs, les trois crues utilisées pour le calage sont des crues fortes. Pour analyser la validité du modèle, une simulation a été effectuée sur une période continue (du 10/05/91 au 5/10/91) pendant laquelle l'évolution des dépôts dans les biefs était observée et ponctuellement mesurée de manière très sommaire (mesure au décamètre d'une longueur, une largeur et une épaisseur moyennes). Pendant cette période les mesures des dépôts dans le piège ont été très correctes, alors que celles des volumes transités ont été plutôt inexistantes. Pour avoir malgré tout une idée de la validité des calculs des matières en suspension, on les a comparés aux volumes transités estimés à partir d'une formule statistique obtenue sur les épisodes antérieurs bien mesurés (BorGEs A.L.,1993). Les graphiques des volumes cumulés sur la période test sont représentés sur les figures 12 et 13 .

On voit que les résultats sont assez satisfaisants. La figure 12 montre que la formule de Rickenmann a tendance à sous-estimer, que les deux autres (mais surtout celle de Lefort) ont peut-être un seuil de début de transport trop faible. Comme les formules de transport ont été utilisées sans aucun calage de paramètre, il semble tout à fait possible de les adapter pour les faire représenter encore mieux les flux sortants du bassin du Laval. 


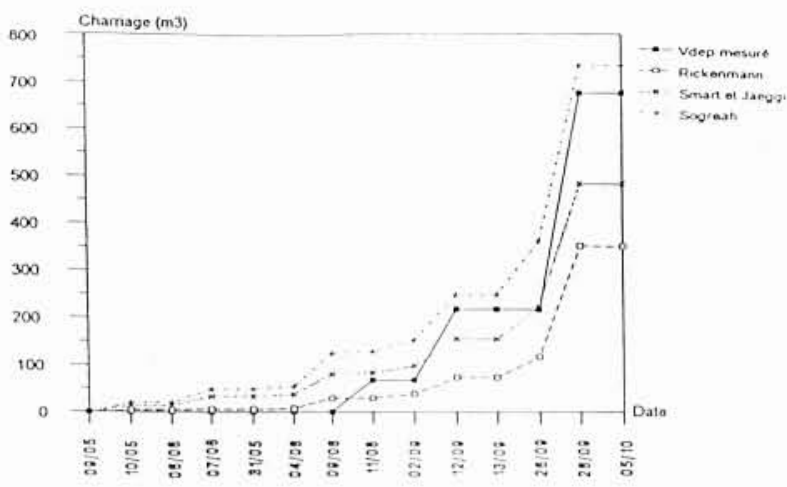

12. Volumes charriés calculés cumulés et volumes déposés mesurés cumulès.

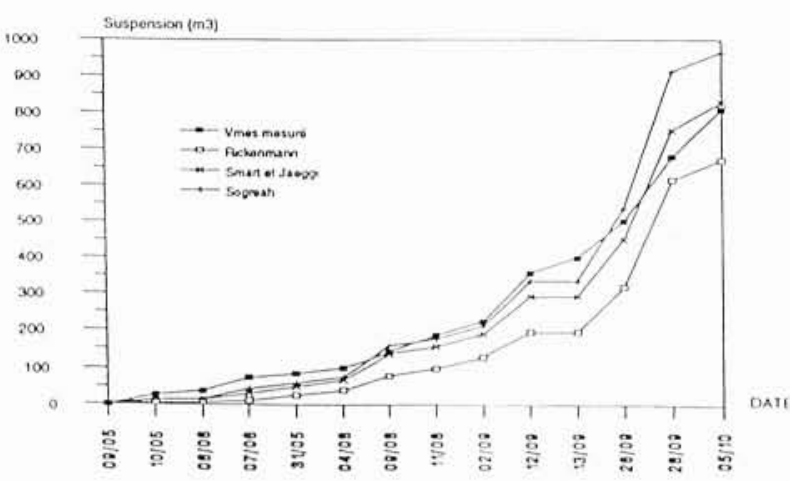

13. Volumes transités estimés cumulés et volumes en suspension calculés cumulés.

Lévolution du stock de sédiments dans les trois biefs, telle qu'elle résulte du calcul, est indiquée sur la figure 14 . Malheureusement, il n'est pas possible de la confronter à des mesures, hormis pour l'origine et la fin de la période ; la forte crue du 28/9/91 ( $97 \mathrm{~mm}$ de pluie) a en effet tout nettoyé. L'accord aux limites est valable, mais on concoit qu'une mesure régulière des dépôts de sédiments dans les biefs aiderait grandement à porter un jugement plus fin sur la qualité de ce modẻle.

\section{Conclusions}

Les principales conclusions de ce travail sont les suivantes :

- Il y a un très bon accord entre les calculs des volumes transportés par charriage et les volumes déposés, et ceci est assez surprenant compte tenu de la nature des matériaux transportés à Draix ; le choix de la meilleure formule n'est pas un problème important: il serait d'ailleurs possible d'adapter une formule aux écoulements hyperconcentrés de Draix, en se donnant un seuil de début de transport plus élevé ;

- La suspension étant très variable d'une crue à l'autre, cela reste un phénomène à mieux observer et mesurer ;

- Néanmoins la dégradation du charriage en suspension, telle qu'elle est proposée dans ce travail, est une voie prometteuse. La valeur proposée du paramètre $k$ de la loi de dégradation, sous-estime vraisemblablement les volumes transités. Elle a été en effet obtenue à partir de valeurs
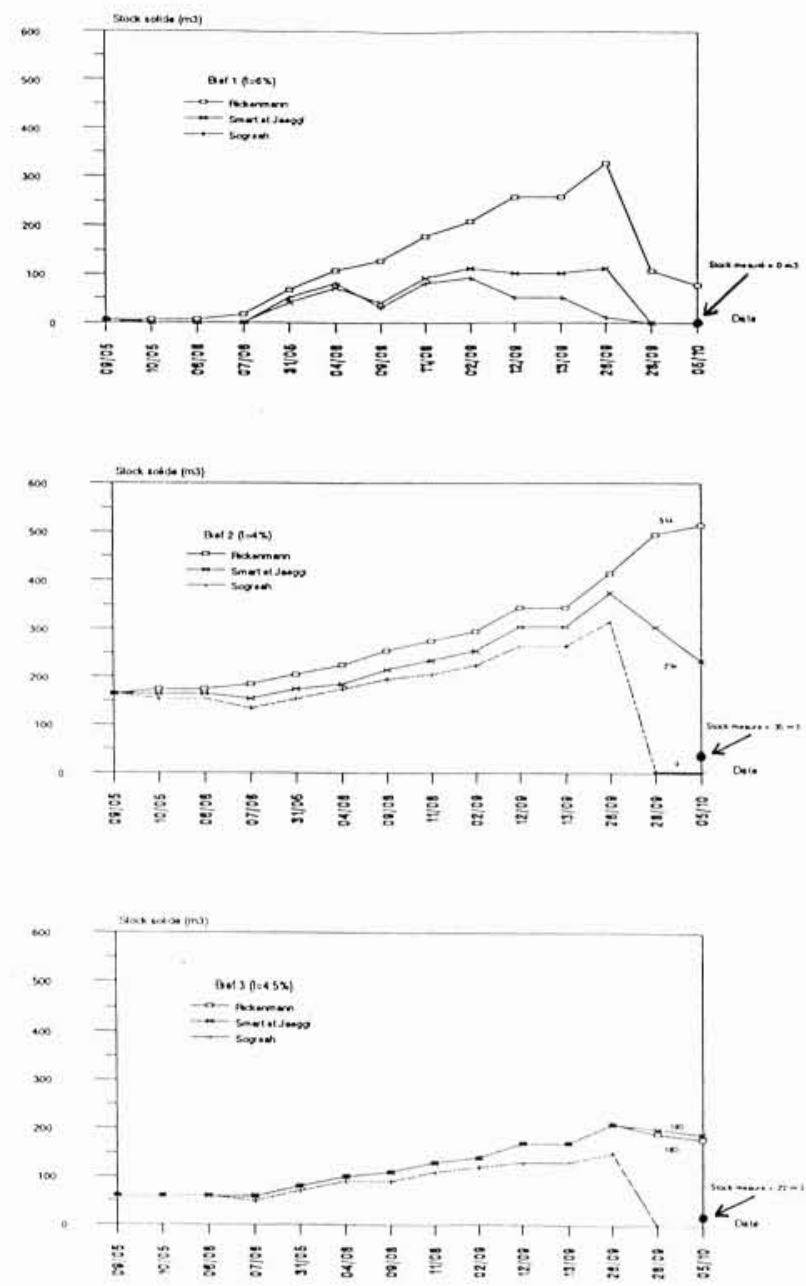

14. Evolution du stock des dépöts dans les biefs du Laval.

annuelles de transport solide, ce qui n'est pas une excellente méthode, étant donné qu'il s'agit d'un phénomène non-linéaire.

Le travail à effectuer en complément pour l'avenir, est donc le suivant :

- Il faut améliorer la mesure des matériaux transités, en installant des appareillages plus adaptés. Sont prévus des appareils basés sur la rétrodiffusion optique, et sur l'absorption par le matériau solide, des rayonnements g obtenus par des sources radioactives;

- La mesure de l'évolution des dépôts dans les biefs est entreprise dans le cadre du projet européen EROSLOPE, en concertation avec ce qui est fait sur d'autres torrents (Bavière, Zürich, Italie du Nord). Elle permettra de les comparer aux volumes calculés; on aura alors une bien meilleure sécurité dans l'estimation de la fiabilité du modèle. Mais, du point de vue du contrôle des variables, restera le problème de la connaissance des entrées dans le modèle hydrosédimentologique; il restera mal résolu, comme c'est toujours le cas dans les problèmes de transport solide torrentiel ;

- On peut enfin étudier la possibilité d'améliorer la finesse du modèle et tenir compte de l'évolution de la morphologie des biefs, en fonction des phénomènes de 
dépôts et de reprise. C'est un problème encore plus difficile que celui qui a été traité ici, et qui nécessiterait une observation de cette morphologie (largeur, pente) pendant la crue elle-même.

\section{Bibliographie}

BATHURST J.C. (1987), - Critical conditions for bed material movement in steep, boulder-bed streams. Corvallis Symposium: Erosion and sedimentation in the Pacific Rim. AIHS Publ. $\mathrm{N}^{*} 165$.

BUFFALO M. (1989). - L'érosion des terres noires dans la région du Büech. Thèse de géologie. Université Aix-Marseille 3.

Borges A.L. (1993). - Modélisation de l'érosion sur deux bassins versants expérimentaux des Alpes du Sud. CEMAGREF. Thèse de l'Université Joseph Fourier. Grenoble.

Cambon J.P., Mathys N., Meunier M., Olivier J.O. (1990). Mesures des débits solides et liquides sur des bassins versants de montagne. Congress Hydrology in Mountainous Regions. Lausanne. AIHS Publ. $N^{\circ} 194$.

Combes F. (1981). - Le barrage du Saignon: un exemple de sédimentation. La gestion régionale des sédiments. Séminaire national. Doc. BRGM N*30. 101-104.

Di Silvio G., Peviani M. (1989). - Modelling short- and longterm evolution of mountain rivers : an application to the torrent Mallero. Int. Workshop on Fluvial Hydraulics of Mountain Regions, Trent, Italy.

Dı SILvıo (1991). - Long-term equilibrium profile of mountain rivers. AIRH Madrid. A503-A510.

Labadie G., Ben Slama E., Chee P., Rahuel J.L. (1991). "Modélisation monodimensionnelle du transport solide en granulométrie étendue. Congrès AIRH. Madrid. A403A411.

LEFORT P. (1992.). - Transport solide dans le lit des cours d'eauDynamique fluviale. ENSHMG-INPG. 167 p.

Lefort P., Meunier M. (1992), - Crues et laves torrentielles. Analyse des phénomènes physiques. Colloque "Crues et laves torrentielles ». Nainville les Roches. DPC-DRMSHF, $29 \mathrm{p}$.

Lefranc P. (1990). - Le Modèle ETC: un modèle d'érosion des torrents en crue. CEMAGREF. PE. ENITRTS. Mémoire de $3^{\mathrm{e}}$ année. $76 \mathrm{p}$.

LiN H. (1993). - Le transport solide en collecteur d'assainissement et sa modélisation. Thèse ENPC. $199 \mathrm{p}$.

MAthys N., MeUnier M. (1989). - « Mesure et interprétation du processus d'érosion dans les marnes des Alpes du Sud à l'échelle de la petite ravine. Colloque transport solide. Nov. 88. SHF Paris. La Houille Blanche $\mathrm{N}^{\circ} 3 / 4.1989$. 188-192.

MEUNIER M. (1987). - Note sur le cadre a priori permettant la mise au point d'un modèle déterministe de simulation de l'érosion et du transport solide sur les petits bassins de montagne. CEMAGREF. Note interne.

MEUNIER M., MATHYS N. (1989). - Etude comparative sommaire des débits de crue des zones urbanisées et des bassins versants naturels en montagne. CEMAGREF/PE Note interne.

Rahuel J.L., Holly F.M., Chollet J.P., Belleudy P., Yang G. (1989). - Modelling of riverbed evolution for bedload sediment mixtures. J. of Hydr. Engrg., ASCE 115(11), $1521-1542$.

Rahuel J.L. (1988). - Modélisation de lévolution du lit des rivières alluvionnaires à gramulométrie étendue. Thèse INPG. Grenoble. 248 p.

RiCKENMAN D. (1990). - Bedload transport capacity of slurry flows at steep slopes. Versuchsanstalt für Wasserbau, Hydrologie und Glaziologie der Eidgenössichen Technischen Hochschule Zürich. Mitteilungen 103.

Smart G.M., JAEGGi M. (1983). - Sediment transport on steep slopes. Zurich, Mitteilung der Versuchsanstalt fur Wasserbau, Hydrologie ud Glaziologie, ETH N 64 .

Zhang J., Huang W.W., Shi M.C. (1990). - Huanghe (Yellow river) and its estuary : sediment origin, transport and deposition. J. of Hydrology, 120 (1990), 203-223. 